\title{
ON THE THEORY OF ONE-SIDED MODELS IN SPACES WITH ARBITRARY CONES
}

\author{
ABSTRACT \\ A. A. Martynyuk and A. Yu. Obolensky \\ Institute of Mechanics \\ The Ukrainian Academy of Sciences \\ 252057, Kiev-57, Nesterov Str. 3 \\ USSR
}

The paper presents a way of constructing quasimonotone nonautonomous systems ensuring $x$-stability of the nonautonomous system. There are described extensions quasimonotone with respect to an arbitrary cone, Perron condition and invariant surface stability under perturbations. Ustability on the set of non-wandering points is proved to imply u-stability of quasimonotone nonlinear system and exponential u-stability on minimal attraction center provides u-stability of the total systems. Examples are available.

Key Words: quasimonotone models, montone extension, u-stability, Perron condition, non-wandering points, minimal attraction center.

AMS Subject Classifications: 34A60, 34D20, 49C15.

\section{INTRODUCTION}

One-side models appeared as a means of investigation of real systems dynamical properties in connection with the application of vector Lyapunov functions the existence of which was indicated by Azbelev [1], Bellman [2], Matrosov [12], Melnikov [14]. The comparison method created hereat is presented in $[4,5,6,9,12,14,18,19]$ and some other papers. In 1977 Lakshmikantham and Leela [7] offered a comparison method for cone-valued Lyapunov functions. The present contribution develops further the ideas of the comparison technique for the case of autonomous and non-autonomous quasimonotone extensions.

In the paper various sufficient conditions for equilibrium state stability in linear and nonlinear models are determined and a relation between the dynamical properties of these models and their behavior on limiting sets established. The concept of "extension" employed allows us to apply the fundamental results from theory of dynamical systems in the investigation of non-autonomous one-side models.

\section{QUASIMONOTONE MODELS IN GENERAL}

Let $\mathrm{B}$ be a compact metric space, $\mathrm{R}^{\mathrm{n}}$ be an $\mathrm{n}$-dimensional Euclidean space. The Cartesian product $B \times R^{n}=E$ with projection $\rho: E \rightarrow B$ is a phase space for the given comparison system. 
Let cone $\mathrm{K}$ with interior $\mathrm{K}$ be given in $\mathrm{R}^{\mathrm{n}}, \mathrm{u}_{1} \geq \mathrm{u}_{2} \stackrel{\text { def }}{=} \mathrm{u}_{1}-\mathrm{u}_{2} \in \mathrm{K}$ for each $\mathrm{u}_{1}, \mathrm{u}_{2}$ $\in \mathrm{R}^{\mathrm{n}},\left\langle\mathrm{u}_{1}, \mathrm{u}_{2}>=\left\{\mathrm{u} \in \mathrm{R}^{\mathrm{n}}: \mathrm{u}_{1} \leq \mathrm{u} \leq \mathrm{u}_{2}\right\}\right.$ is a cone segment, and $\mathrm{K}^{*}$ is a cone conjugated with $\mathrm{K}$. We consider a dynamical system $\mathrm{H}^{\mathrm{t}}: \mathrm{E} \rightarrow \mathrm{E}$.

Definition 2.1. Dynamical system $\mathrm{H}^{\mathrm{t}}$ is called monotone (strictly monotone) extension if

a) $\quad \mathrm{H}^{\mathrm{t}}(\mathrm{v})=\mathrm{H}^{\mathrm{t}}(\rho \mathrm{v})=\mathrm{F}^{\mathrm{t}}(\varphi) \quad$ for each $\quad \mathrm{v}=(\varphi, \mathrm{u}) \in \mathrm{B} \times \mathrm{R}^{\mathrm{n}}$;

b) for each $\left(v_{1}, v_{1}\right) \in$ E such that $\rho\left(v_{1}\right)=\rho\left(v_{2}\right)$ inequalities $v_{1} \geq v_{2}$ and $v_{1} \neq v_{2}$ imply

$$
\mathrm{H}^{\mathrm{t}}\left(\mathrm{v}_{1}\right) \geq \mathrm{H}^{\mathrm{t}}\left(\mathrm{v}_{2}\right) \quad\left(\mathrm{H}^{\mathrm{t}}\left(\mathrm{v}_{1}\right)>\mathrm{H}^{\mathrm{t}}\left(\mathrm{v}_{2}\right)\right) \quad \text { for all } \mathrm{t} \in \stackrel{\mathrm{R}}{\mathrm{R}}_{+} .
$$

The general monotone extension is generated by system

$$
\begin{aligned}
& \mathrm{F}^{\mathrm{t}}: \mathrm{B} \rightarrow \mathrm{B} \\
& \dot{\mathrm{u}}=\mathrm{g}(\varphi, \mathrm{u}), \quad \varphi \in \mathrm{B}, \quad \mathrm{u} \in \mathrm{R}^{\mathrm{n}},
\end{aligned}
$$

where $\mathrm{F}^{\mathrm{t}}$ is a dynamical system in $\mathrm{B}$, which has unique solution $\left(\mathrm{Ft}^{\mathrm{t}}\left(\varphi_{0}\right), \mathrm{u}\left(\mathrm{t} ; \varphi_{0}, \mathrm{u}_{0}\right)\right)$ defined for all $\mathrm{t} \geq 0$ and $\left(\varphi_{0}, \mathrm{u}_{0}\right) \in \mathrm{E}$.

Classes of functions $\mathrm{W}_{0}\left(\mathrm{~W}_{\mathrm{r}}\right)$ [15] that form the montone extensions have the following generators:

1. Functions of the type of $g(\varphi, u)=\alpha(\varphi) u$, where $\alpha: B \rightarrow R$, belong to class $\mathrm{W}_{0}$ (extension of the monotonicity);

2. Convex linear combinations of mappings of the type of

$\mathrm{h}\left(\varphi,\left(\mathrm{u}, \mathrm{z}^{*}(\varphi)\right)\right) \mathrm{z}(\varphi), \quad($, ) is a scalar product, $\mathrm{h}(\varphi, s): \mathrm{B} \times \mathrm{R} \rightarrow \mathrm{R}$ is montone (strictly monotone) increasing with respect to $S \in R$ for all $\varphi \in B$, $\mathrm{z}(\varphi) \in \mathrm{K}$, and $\mathrm{z}^{*}(\varphi) \in \dot{\mathrm{K}}^{*}$, belong to class $\mathrm{W}_{0}\left(\mathrm{~W}_{\mathrm{r}}\right.$ is a strictly monotone extension).

3. Functions of the type of $g(\varphi, u)=A(\varphi) u$, where $A(\varphi)$ is an element of Lie algebra corresponding to Lie group of homogeneous cone $\mathrm{K}$ transformations, belong to class $\mathrm{W}_{0}$.

It should be noted that the class $\mathrm{W}_{0}$ is closed with respect to the limiting transition on compact sets in E.

Definition 2.2. Monotone extension (2.1), (2.2) is called

i) $\mathrm{u}$-stable in cone $\mathrm{K}$ if for any $\varepsilon>0$ there exists $\delta(\varepsilon)>0$ such that $\delta(\varepsilon) \mathrm{e}-\mathrm{u}_{0} \in \mathrm{K}$ for any $\varphi \in \mathrm{M} \subset \mathrm{B}$ implies inclusion $\varepsilon \mathrm{e}-\mathrm{u}\left(\mathrm{t} ; \varphi_{0}, \mathrm{u}_{0}\right) \in \mathrm{K}$ for all $t \in \dot{R}_{+}, \quad e \in \stackrel{\circ}{\mathrm{K}}$; 
ii) asymptotically $\mathrm{u}$-stable in cone $\mathrm{K}$ if it is $\mathrm{u}$-stable in the sense of Definition 2.2.i and for any $\varphi \in B$ a neighborhood $u_{\varphi} \subset R^{n}$ exists such that for $\mathrm{u}_{0} \in \mathrm{u}_{\varphi} \cap \mathrm{K}, \lim \mathrm{u}\left(\mathrm{t} ; \varphi, \mathrm{u}_{0}\right)=0$ takes place as $\mathrm{t} \rightarrow \infty$.

\section{STABILITY AND ASYMPTOTIC BEHAVIOR}

Let $\mathrm{L}^{\mathrm{t}}$ be a dynamical system in $\mathrm{E}=\mathrm{B} \times \mathrm{R}^{\mathrm{n}}$ generated by system of the type of

$$
\begin{gathered}
\mathrm{F}^{\mathrm{t}}: \mathrm{B} \rightarrow \mathrm{B}, \\
\dot{\chi}=\mathrm{f}(\varphi, \chi), \chi \in \mathrm{R}^{\mathrm{m}} .
\end{gathered}
$$

Together with system (3.1), (3.2) we consider a vector-function $\mathrm{V} \in \mathrm{C}\left[\mathrm{B} \times \mathrm{R}^{\mathrm{m}}, \mathrm{K}\right]$, $\mathrm{V}(\varphi, \chi)$ is locally Lipschitzian on $\chi$.

Let there exist a function $\mathrm{Q}(\varphi, \chi): \mathrm{B} \times \mathrm{R}^{\mathrm{m}} \rightarrow \mathrm{K}, \mathrm{Q}(\varphi, 0)=0$, which is non-negative relatively cone $\mathrm{K}$ and function $\mathrm{g} \in \mathrm{C}\left[\mathrm{B} \times \mathrm{K}, \mathrm{R}^{\mathrm{n}}\right], \mathrm{g}(\varphi, \mathrm{u}) \in \mathrm{W}_{0}(\mathrm{~K})$. (3.1), (3.2).

The following assertion is an extension of Theorem 3.1.3 from [8] for system Theorem 3.1. Let system (3.1), (3.2) be such that

i) there exist functions $\mathrm{V}, \mathrm{Q}$, and $\mathrm{g}$ mentioned above;

ii) inequality $\mathrm{D}^{+} \mathrm{V}(\varphi, \chi)+\mathrm{Q}(\varphi, \chi) \leq \mathrm{g}(\varphi, \mathrm{V}(\varphi, \chi))$ takes place for all $(\varphi, \chi) \in \mathrm{B} \times \mathrm{R}^{\mathrm{m}}$;

iii) for all $\mathrm{t} \in \mathrm{R}_{+}$there exists a unique solution of system (2.1), (2.2) when $\mathrm{t} \geq 0$ and $\left(\varphi_{0}, \mathrm{u}_{0}\right) \in \mathrm{E}$.

Then for any solution $\left(\mathrm{F}^{\mathrm{t}}\left(\varphi_{0}\right), \chi\left(\mathrm{t} ; \varphi_{0}, \chi_{0}\right)\right)$ of system (3.1), (3.2) estimate

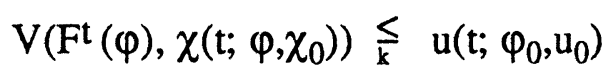

is valid as soon as $\mathrm{v}\left(\varphi_{0}, \chi_{0}\right) \quad \frac{\leq}{\mathrm{k}} \mathrm{u}_{0}$ for each $\varphi_{0} \in \mathrm{B}$.

Theorem 3.2. [15]. If for the monotone extension $H^{t}$ there exists an invariant strictly monotone surface $\Gamma: B \times R \rightarrow E$, then asymptotic behavior of the extension $H^{t}$ on $E$ is defined by its asymptotic behavior on $\Gamma$.

Let strictly monotone extension $\mathrm{H}^{\mathrm{t}}$ be generated by system $\mathrm{Q}$

$$
\mathrm{F}^{\mathrm{t}}: \mathrm{B} \rightarrow \mathrm{B} \text {, }
$$




$$
\dot{\mathrm{u}}=\mathrm{A}(\varphi) \mathrm{u}, \mathrm{u} \in \mathrm{R}^{\mathrm{n}}, \varphi \in \mathrm{B}
$$

Definition 3.1. Strictly monotone extension $\mathrm{H}^{\mathrm{t}}$ satisfies Perron condition if there exists a couple of invariant subspaces $L_{1}(\varphi)$ and $L_{2}(\varphi)$ in $E$ such that $\operatorname{dim}\left(L_{1}(\varphi) \cap \rho^{-1}(\varphi)\right)=1$ where $\rho^{-1}(\varphi)$ is a pre-image of $\rho(\varphi) ; \operatorname{dim}\left(L_{2}(\varphi) \cap \rho^{-1}(\varphi)\right)=n-1$;

$$
\begin{aligned}
& \mathrm{L}_{1}(\varphi) \subset(\stackrel{\mathrm{K} \cup}{\mathrm{K}} \stackrel{\mathrm{K}}{ }) \cap \rho^{-1}(\varphi) ; \\
& \mathrm{L}_{2}(\varphi) \cap(\mathrm{K} \backslash 0) \cap \rho^{-1}(\varphi)=\varnothing
\end{aligned}
$$

and constants $\mathrm{c}>0$ and $0<\delta<1$ exist such that

$$
\frac{\left\|\mathrm{H}_{\varphi}^{\mathrm{t}}\left(\mathrm{v}_{2}\right)\right\|}{\left\|\mathrm{v}_{2}\right\|} / \frac{\left\|\mathrm{H}_{\varphi}^{\tau}\left(\mathrm{v}_{1}\right)\right\|}{\left\|\mathrm{v}_{1}\right\|} \leq \mathrm{c} \delta^{\mathrm{t}} \quad \text { for each } \mathrm{t} \geq 0 .
$$

Here $\mathrm{v}_{\mathrm{i}}=\left(\varphi, \mathrm{u}_{\mathrm{i}}\right), \mathrm{u}_{\mathrm{i}} \neq 0, \mathrm{u}_{\mathrm{i}} \in \mathrm{L}_{\mathrm{i}}(\varphi), \mathrm{i}=1,2$.

Lemma 3.1. Strictly monotone extension $H^{t}$ satisfies the Perron condition if mapping $A(\varphi) u$ is continuous, bounded, and strictly quasimonotone relatively homogeneous cone $K$.

Theorem 3.3. If linear approximation (3.4), (3.5) of quasilinear extension

$$
\begin{gathered}
\mathrm{F}^{\mathrm{t}}: \mathrm{B} \rightarrow \mathrm{B}, \\
\dot{\mathrm{u}}=\mathrm{A}(\varphi) \mathrm{u}+\mu \mathrm{f}(\varphi, \mathrm{u})
\end{gathered}
$$

satisfies the Perron condition, $f(\varphi, u)$ satisfies the Lipschitz condition, $f(\varphi, 0)=0$ and is quasimonotone on u relatively cone $K$, there exists $\mu_{0}>0$ such that for $0<\mu<\mu_{0}$ extension (3.6), (3.7) has monotone invariant surface $\Gamma$. Proof. For the proof see $[15,16]$.

Further we designate by $\mathrm{Z}$ a set of nonwandering points of dynamical system $\mathrm{F}^{\mathrm{t}}$ and by $\mathrm{C}_{+}$a minimal attraction center of this system.

Theorem 3.4. Let $H^{t}: E \rightarrow E$ be a monotone extension over a compact metric space $B$ and there exist an open in $E$ neighborhood $U$ of a set of nonwandering points $Z$ belonging to dynamical system $\rho H^{t}(\chi)=H^{t}(\rho(\chi))=F^{t}$ such that for $\chi \in U$ and $\rho(\chi) \in Z$, $\left|H^{t}(\chi)-\rho H^{t}(\chi)\right| \Rightarrow 0$ as $t \rightarrow+\infty$. Then for any $\varphi \in B$ there exists a neighborhood $V(\varphi) \subset E$ such that for any $\chi \in V(\varphi) \cap K \cap \rho^{-1}(\varphi),\left|H^{t}(\chi)-\rho H^{t}(\chi)\right| \rightarrow 0$ takes place as $t \rightarrow+\infty, H^{t}(\chi)$ is asymptotically stable for $t \rightarrow+\infty$. 
Proof. Let us set $\mathrm{u}_{1} \in \mathrm{K}^{\circ}$ arbitrary and consider element $\mathrm{u}_{1}(\varphi)=\lambda(\varphi) \mathrm{u}_{1}$ in space $\mathrm{E}$, $\lambda(\varphi)>0$ and $u_{1} \in U$ for all $\varphi \in Z \subset B$. Since the set of nonwandering points $Z$ is compact, there exists $\lambda_{1}=$ const $>0$ such that constant $u(\varphi)=\lambda_{1} \times u_{1}=u$, belongs to $U$ for all $\varphi \in Z$. As the set $\mathrm{Z}$ is invariant and by hypotheses of the theorem there exists a sequence $\left\{T_{0 i}\right\}, i=1,2, \ldots$ such that

for all $\mathrm{t} \geq \mathrm{T}_{0 \mathrm{i}}$.

$$
0 \leq \mathrm{H}_{\varphi}^{\mathrm{t}}(\mathrm{u}) \leq \frac{\mathrm{u}}{8 \cdot 2^{\mathrm{i}}}, \varphi \in \mathrm{Z}, \mathrm{i}=1,2, \ldots
$$

As the dynamical system $\mathrm{H}^{\mathrm{t}}: \mathrm{E} \rightarrow \mathrm{E}$ is continuous, there exist neighborhoods $\mathrm{W}_{\mathrm{i}} \subset \mathrm{B}$ of $\operatorname{set} \mathrm{Z}$ in $\mathrm{B}$ such that estimate

$$
0 \leq \mathrm{H}_{\varphi}^{\mathrm{T}_{0 \mathrm{i}}}(\mathrm{u}) \leq \frac{\mathrm{u}}{4 \cdot 2^{\mathrm{i}}}, \varphi \in \mathrm{W}_{\mathrm{i}}, \mathrm{i}=1,2, \ldots
$$

holds for all $\varphi \in \mathrm{W}_{\mathrm{i}}$.

We note that $\mathrm{W}_{\mathrm{i}+1} \subset \mathrm{W}_{\mathrm{i}}$. Let $\mathrm{D}_{\mathrm{i}} \subset \mathrm{W}_{\mathrm{i}} \subset \mathrm{B}$ be a set of points $\varphi \in \mathrm{W}_{\mathrm{i}}$ for which $\mathrm{t} \geq 0$ and $\mathrm{F}^{\mathrm{t}}(\varphi) \in \mathrm{W}_{\mathrm{i}}$. Sets $\mathrm{D}_{\mathrm{i}}$ are nonempty because any point from $\mathrm{B}$ is wandering out of $\mathrm{W}_{\mathrm{i}}$ for a finite time only; $Z \subset D_{i}$ and closures $\bar{D}_{i}$ are compact.

Let $\mathrm{g}_{1}=\underset{\substack{\varphi \in \overline{\mathrm{D}}_{1}^{1} \\ 0 \leq \mathrm{t} \leq \mathrm{T}_{01}}}{\bigcup}<0, \mathrm{H}_{\varphi}^{\mathrm{t}}(\mathrm{u})>$. Set $\mathrm{g}_{1}$ is closed compact and as extension $\mathrm{H}^{\mathrm{t}}: \mathrm{E} \rightarrow \mathrm{E}$ is monotone, $\mathrm{g}_{1}$ is positive invariant. Thus, trajectory $\mathrm{H}^{\mathrm{t}}(\chi)$ is positive stable in the sense of Lagrange for every $\chi \in \mathrm{E}$ and $\chi \in \mathrm{g}_{1}$.

By choice of sequence $\mathrm{T}_{0 \mathrm{i}}$ and monotonicity of extension $\mathrm{H}^{\mathrm{t}}: \mathrm{E} \rightarrow \mathrm{E}$ inequality

$$
0 \leq \mathrm{H}_{\varphi}^{\mathrm{mT}}(\mathrm{u}) \leq \frac{\mathrm{l}}{4 \cdot 2^{\mathrm{i}}}, \mathrm{i}=1,2, \ldots
$$

takes place for $\varphi \in \bar{D}_{1}$, where $m$ is a positive integer.

For an arbitrary $\varphi \in \overline{\mathrm{D}}_{\mathrm{i}}$ we can take $\mathrm{m}(\varphi, \mathrm{i})$ such that correlation

$$
0 \leq \mathrm{H}_{\varphi}^{\mathrm{t}}(\mathrm{u}) \leq \frac{\mathrm{u}}{2 \cdot 2^{\mathrm{i}}}, \mathrm{i}=1,2, \ldots
$$


holds true for all $\mathrm{t} \in\left[(\mathrm{m}(\varphi, \mathrm{i})+1) \mathrm{T}_{0 \mathrm{i}},(\mathrm{m}(\varphi, \mathrm{i})+2) \mathrm{T}_{0 \mathrm{i}}\right]$.

Suppose on the contrary. Then sequence $\mathrm{m}_{l}(\varphi, \mathrm{i}) \rightarrow+\infty$ as $l \rightarrow \infty$ and numbers $T_{l} \in\left[\mathrm{T}_{0 \mathrm{i}}, 2 \mathrm{~T}_{0 \mathrm{i}}\right]$ exist such that

$$
\mathrm{H}^{\mathrm{m}_{\mathrm{l}}(\varphi, \mathrm{i}) \mathrm{T}} \text { oi }(\mathrm{u}) \leq \frac{\mathrm{u}}{4 \cdot 2^{\mathrm{i}}}, \quad \mathrm{H}_{\varphi}^{\mathrm{m}_{\mathrm{l}}(\varphi, \mathrm{i}) \mathrm{T}_{0 \mathrm{i}}+\tau_{\mathrm{l}}} \quad(\mathrm{u}) \notin\left\langle 0, \frac{\mathrm{u}}{2 \cdot 2^{\mathrm{i}}}\right\rangle .
$$

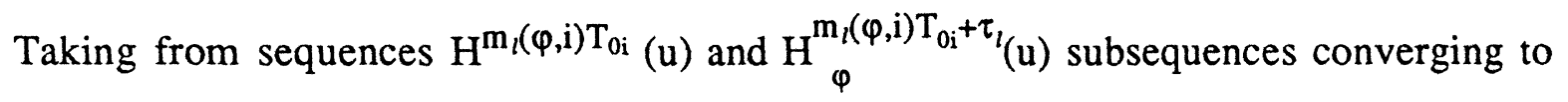
some $u_{\infty}$ and $v_{\infty}, \rho\left(u_{\infty}\right) \in Z$ and $\rho\left(v_{\infty}\right) \in Z$, we make sure that $u_{\infty} \in\left\langle 0, \frac{v}{4 \cdot 2^{\mathrm{i}}}\right\rangle$, $\mathrm{v}_{\infty} \notin\left\langle 0, \frac{\mathrm{u}}{2 \cdot 2^{\mathrm{i}}}\right\rangle$. Then $\mathrm{a} \tilde{\mathrm{t}} \in\left[\mathrm{T}_{0 \mathrm{i}}, 2 \mathrm{~T}_{0 \mathrm{i}}\right]$ exists such that

$$
\mathrm{H}^{\tilde{t}}\left(\mathrm{u}_{\infty}\right)=\mathrm{v}_{\infty}
$$

Equality (3.9) contradicts to the choice of $\mathrm{T}_{0 \mathrm{i}}$ and to inequality (3.8). Since

$$
0 \leq \mathrm{H}_{\varphi}^{\mathrm{t}}(\mathrm{u}) \leq \frac{\mathrm{u}}{2 \cdot 2^{\mathrm{i}}}<\mathrm{u}, \mathrm{i}=1,2, \ldots, \varphi \in \mathrm{D}_{\mathrm{i}},
$$

for all $\mathrm{t} \in\left[(\mathrm{m}(\varphi, \mathrm{i})+1) \mathrm{T}_{0 \mathrm{i}},(\mathrm{m}(\varphi, \mathrm{i})+2) \mathrm{T}_{0 \mathrm{i}}\right]$, then applying operator $\mathrm{H}^{/ \mathrm{T}_{0 \mathrm{i}}}$ to inequality (3.10) we obtain by virtue of the extension monotonicity

$$
0 \leq \mathrm{H}_{\varphi}^{\mathrm{t}}(\mathrm{u}) \leq \frac{\mathrm{ll}}{2 \cdot 2^{\mathrm{i}}}, \varphi \in \overline{\mathrm{D}}_{\mathrm{i}}, \mathrm{i}=1,2, \ldots,
$$

for all $\mathrm{t} \geq(\mathrm{m}(\varphi, \mathrm{i})+1) \mathrm{T}_{0 \mathrm{i}}$.

As closure $\bar{D}_{1}$ is compact, the choice of $m(\varphi, i)$ can be made independently of $\varphi \in D_{i}$, where inequality (3.11) ensures correlation

for all $\mathrm{t} \geq \mathrm{m}(\mathrm{i})$.

$$
0 \leq \mathrm{H}_{\varphi}^{\mathrm{t}}(\mathrm{u}) \leq \frac{\mathrm{u}}{2^{\mathrm{i}}}, \varphi \in \overline{\mathrm{D}}_{\mathrm{i}}, \mathrm{i}=1,2, \ldots
$$

Let $T_{i j}(\varphi), i<j$, be a wandering time of arbitrary points $\varphi \in D_{i}$ out of $W_{j}$. Then the arguments above imply that inequality

holds for any $t \geq m(j) T_{0 j}+T_{i j}+m(i) T_{0 i}$.

$$
0 \leq \mathrm{H}_{\varphi}^{\mathrm{t}}(\mathrm{u}) \leq \frac{\mathrm{u}}{2^{\mathrm{j}}}, \varphi \in \mathrm{D}_{\mathrm{i}}
$$

Thus, $\mathrm{H}_{\varphi}^{\mathrm{t}}(\mathrm{u}) \rightarrow 0$ as $\mathrm{t} \rightarrow+\infty$ and $\varphi \in \mathrm{D}_{1}$. 
As the extension is monotone for any $\chi \in E, \rho(\chi) \in D_{1}, \chi=(\varphi, y)$ and $y \in\langle 0, u\rangle$, then $\left|\mathrm{H}^{\mathrm{t}}(\chi)-\rho \mathrm{H}^{\mathrm{t}}(\chi)\right| \rightarrow 0$ as $\mathrm{t} \rightarrow+\infty$.

We set $\varphi \in B$ arbitrary and take $T>0$ such that $\mathrm{F}^{\mathrm{T}}(\varphi) \in \mathrm{D}_{1}$. The required neighborhood $\mathrm{V}(\varphi)$ exists as a pre-image of cone segment $\langle 0, u\rangle^{\bullet}$ for mapping $\mathrm{H}_{\mathrm{F}^{\mathrm{T}}(\varphi)}^{-\mathrm{T}}$

Theorem 3.5. Let in system (3.4), (3.5)

a) matrix $\mathrm{A}(\varphi) \mathrm{u}$ be continuous bounded and quasimonotone relatively cone $K$;

b) there exist an invariant subspace $\mathrm{L}_{1}(\varphi): \operatorname{dim}\left(\mathrm{L}_{1}(\varphi) \cap \rho^{-1}(\varphi)\right)=1$, $\mathrm{L}_{1}(\varphi) \cap \stackrel{\circ}{\mathrm{K}} \neq \varnothing$ with respect to $\mathrm{H}^{\mathrm{t}}$;

c) trajectories $\rho\left(\mathrm{H}^{+}(\mathrm{v})\right), \mathrm{v} \in \mathrm{E}$ be exponentially $u$-stable for $\rho(\mathrm{v}) \in \mathrm{C}_{+}$.

Then $H^{t}$ is exponentially $u$-stable in cone $K$.

Proof. Due to the continuity of $\mathrm{L}_{1}$ and its differentiability along system $\mathrm{F}^{+}(\varphi)$ trajectories, the system (3.4), (3.5) on $\mathrm{L}_{1}$ is generated by a system of the form

$$
\begin{aligned}
& \mathrm{Ft}^{\mathrm{t}}: \mathrm{B} \rightarrow \mathrm{B} \\
& \mathrm{s}=\mathrm{g}(\varphi) \mathrm{s}, \mathrm{s} \in \mathrm{R}, \varphi \in \mathrm{B}
\end{aligned}
$$

with continuous bounded function $\mathrm{g}: \mathrm{B} \rightarrow \mathrm{R}$.

As B is compact and system (3.4), (3.5) is linear, it is enough to show that there exists a $\beta<0$ such that

$$
\varlimsup_{t \rightarrow+\infty} \frac{1}{t} \int_{0}^{t} g\left(F^{\tau}(\varphi)\right) d \tau \leq \beta
$$

for all $\varphi \in \mathrm{B}$.

Let us set $\varphi \in B$ arbitrary and find a sequence $T_{l} \rightarrow \infty$ as $l \rightarrow \infty$ such that

$$
\frac{1}{T_{l}} \int_{0}^{T_{l}} g\left(F^{\tau}(\rho)\right) d \tau \rightarrow \varlimsup_{t \rightarrow+\infty} \frac{1}{t} \int_{0}^{t} g\left(F^{\tau}(\varphi)\right) d \tau .
$$

Let $\mathrm{m}_{\varphi}(\mathrm{u})$ be a normed measure concentrated at $\varphi \in \mathrm{B}$. For an arbitral function $\mathrm{f}: \mathrm{B} \rightarrow \mathrm{R}$ we define a sequence of measures by equality 


$$
\int_{B} f(\psi) m_{\varphi T_{i}}(d \psi)=\frac{1}{T_{l}} \int_{0}^{T_{i}} d \tau \int_{B} f\left(F^{\tau}(\varphi)\right) d m_{\varphi}(u) .
$$

By definition of $\mathrm{m}_{\varphi}(\mathrm{u})$

$$
\int_{B} f(\psi) m_{Q, T_{i}}(d \psi)=\frac{1}{T_{i}} \int_{0}^{T_{i}} f\left(F^{\tau}(\varphi)\right) d \tau .
$$

From the sequence of measures $\mathrm{m}_{\varphi, \mathrm{T}_{l}}$ we take a subsequence weakly convergent to some limiting measure $\mu_{\varphi}(u)$.

For the limiting measure $\mu_{\varphi}(\mathfrak{u})$ there exists a sequence of measures $\left\{\mu_{\psi_{i}}\right\}$, $\mu_{\Psi_{i}} \in \Sigma_{\mu}$, of fundamental system of measures and numbers $\lambda_{\mathrm{i}}, \sum_{\mathrm{i}=1}^{\mathrm{m}} \lambda_{\mathrm{i}}=1$, such that measures $\sum_{i=1}^{m} \lambda_{i} \mu_{\psi_{i}}$ are weakly convergent to the measure $\mu_{\varphi}(u)$ for $m \rightarrow \infty$, and equalities

take place.

$$
\begin{aligned}
\int_{B} g(\psi) \mu_{\varphi}(d \psi) & =\lim _{t \rightarrow \infty} \frac{1}{t} \int_{0}^{t} g\left(F^{\tau}(\varphi)\right) d \tau \\
& =\lim _{m \rightarrow \infty} \sum_{i=1}^{m} \int_{B} g(\psi) \mu_{\psi_{1}}(d \psi)
\end{aligned}
$$

There exists a constant $\beta<0$ such that for any $\mu_{\psi_{i}} \in \Sigma_{\mu \varphi}, \int_{B} g(\psi) \mu_{\psi_{i}}(d \psi) \leq \beta$. In fact, assuming on the contrary we find a sequence of invariant normed transitive measures $\mu_{\mathrm{j}}$, $\mu_{\mathrm{j}} \in \Sigma_{\mu}$, such that $\lim _{\mathrm{j} \rightarrow \infty} \int_{\mathrm{B}} \mathrm{g}(\psi) \mu_{\mathrm{j}}(\mathrm{d} \psi) \geq 0$.

The weak limit of measures $\mu_{\mathrm{j}}$ is the measure $\mu_{0}$ which is normed transitive and invariant for dynamical system $\mathrm{Ft}^{\mathrm{t}}(\varphi)$. Choosing $\psi_{0} \in \mathrm{C}_{+}$so that $\psi_{0}$ is regular with respect to measure $\mu_{0}$ we make sure that

$$
\int_{B} g(\psi) \mu_{0}(d \psi)=\lim _{t \rightarrow \infty} \frac{1}{t} \int_{0}^{t} g\left(F^{\tau}\left(\psi_{0}\right)\right) d \tau \geq 0 .
$$

The inequality obtained contradicts to the exponential $U$-stability of $C_{+}$. In the view of the extension monotonicity the theorem is proved.

Let the comparison system be of the form 


$$
\dot{\mathrm{u}}=\mathrm{g}(\mathrm{u})
$$

where $u \in R^{n}, g \in C\left[K, R^{n}\right]$ and $g(u) \in W_{0}(K)$.

The following assertion is the development of results obtained in $[3,10,15]$.

Theorem 3.6. State $\mathrm{u}=0$ of system (3.8) is

a) asymptotically stable in $\mathrm{K}$ if and only if there exists $a \mathrm{c} \in \stackrel{\circ}{\mathrm{K}}$ such that the system of inequalities

has solutions in cone $\mathrm{K}$;

$$
\mathrm{g}(\mathrm{c})<0
$$

b) stable in cone $\mathrm{K}$ if there exists a sequence $\left\{\mathrm{c}_{\mathrm{k}}\right\}$ in $\mathrm{K}^{\circ}, \mathrm{c}_{\mathrm{k}}>0$ and $\mathrm{c}_{\mathrm{k}} \downarrow 0$, for $\mathrm{k} \rightarrow \infty$ such that

for all $\mathrm{k}$;

$$
g\left(c_{k}\right)=0
$$

c) unstable in cone $\mathrm{K}$ if there exists a sequence $\left\{\mathrm{r}_{\mathrm{k}}\right\}$ in $\stackrel{\mathrm{K}}{\mathrm{K}}, \mathrm{r}_{\mathrm{k}} \geq 0$ and $\mathrm{r}_{\mathrm{k}} \downarrow 0$, for $\mathrm{k} \rightarrow \infty$ such that for all $\mathrm{k}, \mathrm{g}\left(\mathrm{r}_{\mathrm{k}}\right) \in \mathrm{K} \backslash 0$ and sets $\mathrm{K}_{\mathrm{c}_{\mathrm{k}}} \cap \mathrm{U}$ do not contain singular points of system (3.8).

Here $\mathrm{K}_{\mathrm{r}_{\mathrm{k}}}=\left\{\mathrm{r} \in \mathrm{R}^{\mathrm{n}}, \mathrm{r}>\mathrm{r}_{\mathrm{k}}\right\}$ and $\mathrm{U}$ is a neighborhood of state $\mathrm{u}=0$.

\section{APPLICATION}

Theorem 3.1 establishes the relation between dynamical properties of solution of equations (3.1), (3.2) and dynamical properties of solutions of one-side model (2.1), (2.2) similarly to the method of comparison with scalar and vector Lyapunov functions (see [15] and References to Chapter II).

Example 4.1. We consider a problem on $\chi$-stability of system

$$
\begin{aligned}
& \dot{u}_{1}=\left(u_{1}^{2}+u_{2}^{2}\right) v_{1} ; \\
& \dot{v}_{1}=-\left(u_{1}^{2}+u_{2}^{2}\right) u_{1} ; \\
& \dot{u}_{2}=\lambda\left(u_{1}^{2}+u_{2}^{2}\right) v_{2} ; \\
& \dot{v}_{2}=-\lambda\left(u_{1}^{2}+u_{2}^{2}\right) u_{2} ;
\end{aligned}
$$

$$
\dot{\chi}=\mathrm{A}\left(\mathrm{u}_{1}, \mathrm{u}_{2}, \mathrm{v}_{1}, \mathrm{v}_{2}\right) \chi,
$$


where $\chi \in R^{n}, \quad\left(u_{1}, v_{1}\right), \quad\left(u_{2}, v_{2}\right) \in R^{2}, \quad$ and $A \in C\left[R^{2} \times R^{2}, R^{n}\right]$ and is bounded. System (4.1a) has a family of invariant tori of the form $u_{1}^{2}+v_{1}^{2}=r_{1}^{2}, u_{2}^{2}+v_{2}^{2}=r_{2}^{2}$,

$\mathrm{r}_{1}, \mathrm{r}_{2},>0$ - const.

System (4.1) in polar coordinates has the form

$$
\begin{gathered}
\dot{\mathrm{r}}_{1}=0, \quad \dot{\mathrm{r}}_{2}=0 ; \\
\dot{\varphi}_{1}=-\left(\mathrm{r}_{1}^{2} \cos ^{2} \varphi_{1}+\mathrm{r}_{2}^{2} \cos ^{2} \varphi_{2}\right) ; \\
\dot{\varphi}_{2}=-\lambda\left(\mathrm{r}_{1}^{2} \cos ^{2} \varphi_{1}+\mathrm{r}_{2}^{2} \cos ^{2} \varphi_{2}\right) ; \\
\dot{\chi}=\mathrm{A}\left(\mathrm{r}_{1}, \mathrm{r}_{2}, \varphi_{1}, \varphi_{2}\right) \chi .
\end{gathered}
$$

Let us consider in $\frac{\mathrm{n}(\mathrm{n}+1)}{2}$-dimensional space a monotone extension induced by mapping $\mathrm{W}=\left[\mathrm{w}_{\mathrm{ij}}\right]=\left[\chi_{\mathrm{i}} \cdot \chi_{\mathrm{j}}\right](\mathrm{i}, \mathrm{j}) \in[1, \mathrm{n}]$, reducing system $(4.2 \mathrm{~b})$ to the differential Lyapunov equation

$$
\dot{\varphi}_{1}=-\left(r_{1}^{2} \cos ^{2} \varphi_{1}+r_{2}^{2} \cos ^{2} \varphi_{2}\right)
$$

We transform (4.3b) to the following

$$
\dot{\varphi}_{2}=-\lambda\left(r_{1}^{2} \cos ^{2} \varphi_{1}+r_{2}^{2} \cos ^{2} \varphi_{2}\right)
$$

where $($,$) is a scalar product of matrices (W, E)=s \rho W E, E$ is a unique matrix.

For $\mu>0$ equation (4.4b) majorizes equation (4.3b) and generates strictly monotone extension relatively cone of positive definite symmetric matrices. Therefore we can apply Lemma 3.1 and Theorem 3.5 to system (4.4b).

For system (4.3a)

$$
\mathrm{C}_{+}=\left\{\stackrel{*}{\varphi_{1}}=\frac{\pi}{2}+\mathrm{k} \pi ; \quad \varphi_{2}^{*}=\frac{\pi}{2}+\mathrm{k} \pi, \mathrm{k} \text { is an integer }\right\}
$$


According to Theorem 3.6 one can observe exponential W-stability in system (4.3) if the system of inequalities

$$
A W_{0}+W_{0} A^{T}+\mu\left(W_{0}, E\right) E<0
$$

has solutions on $\mathrm{C}_{+}$. Due to the definition of $\mathrm{W}$ we find that condition (4.5) is equivalent to Routh-Hurwitz condition on $\mathrm{C}_{+}$for system (4.1) when $\chi$-stability is under consideration.

Example 4.2. Let in an isodromic control system

$$
\begin{gathered}
\frac{d \chi_{i}}{d t}=-\rho_{i} \chi_{i}+a_{i} \sigma, \\
\frac{d \sigma}{d t}=a_{1} \chi_{1}-\sum_{i=2}^{n} a_{i} \chi_{i}-\rho_{n+1} \sigma-f(\sigma)
\end{gathered}
$$

the conditions $0<\rho_{1}<\min _{k}\left(\rho_{k}\right), k \in[2, n+1], \sigma f(\sigma)>0$, for each $\sigma \neq 0, f(0)=0$, $\sigma_{i}^{k}>0$ be fulfilled

Remark 4.1. In contrast to the classical statement of the problem on stability of isodromic control system here we take into account the signs before coefficients $a_{i}$ and this allows us to separate from the general class of isodromic control systems those generating quasimonotone semi-groups. Also it should be noted that the sign of coefficients $a_{i}$ are really different when the real automatic control systems are under consideration.

Systems of equations (4.7) generate quasimonotone semi-groups with respect to a circular cone $\mathrm{K}=\left\{\left(\chi_{\mathrm{i}}, \sigma\right) \in \mathrm{R}^{\mathrm{n}+1}: \chi_{1} \geq 0, \chi_{1}^{2} \geq \sum_{\mathrm{i}=2}^{\mathrm{n}} \chi_{\mathrm{i}}^{2}+\sigma^{2}\right\}$.

The zero solution of (4.7) is uniformly asymptotically stable if and only if the system of inequalities

$$
\begin{aligned}
-\rho_{1} \chi_{1}+a_{1} \sigma & <0, \\
\left(-\rho_{1} \chi_{1}+a_{1} \sigma\right)^{2} & \geq \sum_{i=2}^{n}\left(-\rho_{i} \chi_{i}+a_{i} \sigma\right)^{2}+\left(\sum_{i=2}^{n}\left(a_{i} \chi_{i}-\rho_{n+1} \sigma-f(\sigma)\right)^{2},\right. \\
\chi_{1} & \geq 0 \\
\chi_{1}^{2} & \geq \sum_{i=2}^{n} \chi_{i}^{2}+\sigma^{2}
\end{aligned}
$$

has solutions. This is valid if and only if

$$
\rho_{n+1}+\sum_{i=2}^{n} \frac{a_{1}^{2}}{\rho_{1}}>\frac{a_{1}^{2}}{\rho_{1}}-\frac{f(\sigma)}{\sigma}
$$


for $\sigma \neq 0$.

Remark 4.2. In [10] we established sufficient conditions for uniform asymptotic stability as follows

$$
\rho_{n+1} \geq \sum_{i=1}^{n} \frac{a_{i}^{2}}{\rho_{i}} .
$$

where the signs before coefficients $\mathrm{a}_{\mathrm{i}}$ were not taken into account.

It is easily seen that condition on parameters (4.7) extends (4.8).

\section{CONCLUDING REMARKS}

The consideration of comparison system as an extension of dynamical system defined on compact manifold allows

a) more detailed investigation of linear non-autonomous systems;

b) more complete collection of dynamical properties of comparison and initial system, such as, for example, oscillating processes.

\section{REFERENCES}

[1] Azbelev, N. V. On Chaplygin's problem, Dissertation paper, Izhevsk (1962) (Russian).

[2] Bellman, R. Vector Lyapunov functions, J. Soc. Industr. and Appl. Math., Ser A, Control, vol. 1, 1 (1962) 32-34.

[3] Burgat, C., J. Bernusson, Lj. T. Grujið̌, P. Borne, and J. C. Gentina. Les perturbations structurelles arbitraires et periodiques, RAIRO Automat./Syst. Anal. und Contr., vol. 12, 3, 245-267 (1978).

[4] Grujǐ̌, Lj. T. Stabilnost velikih systems, Beograd Univ. (1974).

[5] Gruji乏, Lj. T., A. A. Martynyuk, and M. Ribbens-Pavella. Large scale systems stability under structural and singular perturbations, Springer-Verlag (1987).

[6] Lakshmikantham, V., and S. Leela. Differential and integral inequalities, Theory and Applications, Volumes. I, II, N.Y., Acad. Press (1969).

[7] Lakshmikantham, V., and S. Leela. Cone valued Lyapunov functions, Nonlin. Anal., 1, 215-222 (1977). 
[8] Lakshmikantham, V., S. Leela, and A. A. Martynyuk. Stability analysis of nonlinear systems, Marcel Dekker (1988).

[9] Martynyuk, A. A. Motion stability of complex systems, Kiev, Nauk. Dumka Publ. (1975) (Russian).

[10] Martynyuk, A. A., and A. Yu.Obolensky. On stability of autonomous Wazewskii's systems solutions, Diff. Uravnenia, 8, 1392-1407 (1980) (Russian).

[11] Matrosov, V. M. On motion stability, Appl. Math. and Mech., vol. 26, 6, 992 1002 (1962) (Russian).

[12] Matrosov, V. M., L. Yu. Anapolsky, and S. N. Vasiliev. Comparison method in mathematical systems theory, Novosibirsk (1980) (Russian).

[13] Melnikov, G. I. Nonlinear mechanical and electromechanical systems motion, Leningrad (1975) (Russian).

[14] Michel, A. N., and R. K. Miller. Qualitative analysis of large scale dynamical systems, N.Y., Acad. Press (1977).

[15] Obolensky, A. Yu. Dynamical systems in space with cone, Kiev (1986). Dep. in VINITI N 7503-B86 (Russian).

[16] Obolensky, A. Yu. Invariant manifolds of dynamical systems in spaces with cone, Kiev (1986). Dep. in VINITI N 7502-B86 (Russian).

[17] Obolensky, A. Yu. Asymptotic behaviour of dynamical systems in spaces with cone, Kiev (1986). Dep. in VINITI N 7504-B86 (Russian).

[18] Rouche, N., P. Habets and M. Laloy. Lyapunov's direct method in stability theory, Moscow, Mir Publ. (1980).

[19] Siljak, D. D. Large scale dynamical systems: stability and structure, N.Y., North Holland (1978). 


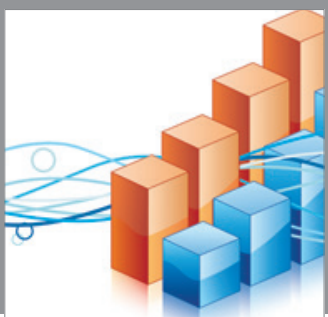

Advances in

Operations Research

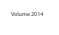

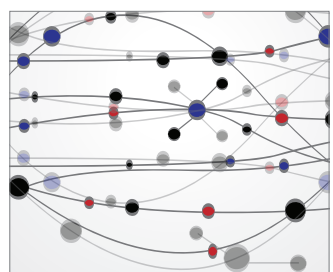

\section{The Scientific} World Journal
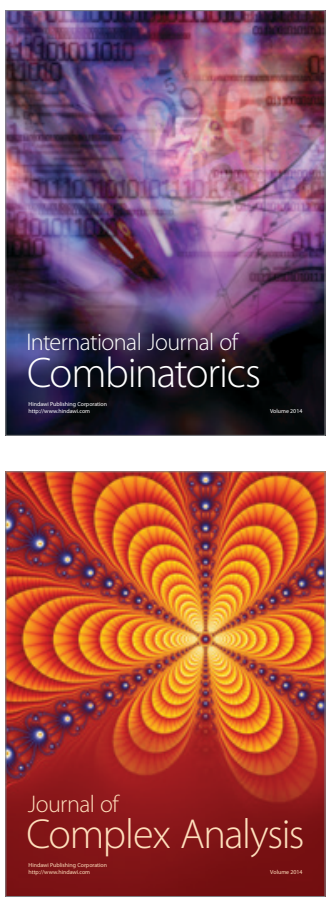

International Journal of

Mathematics and

Mathematical

Sciences
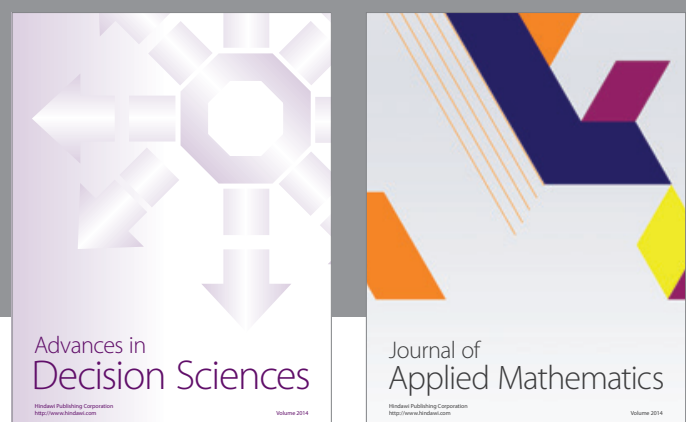

Journal of

Applied Mathematics
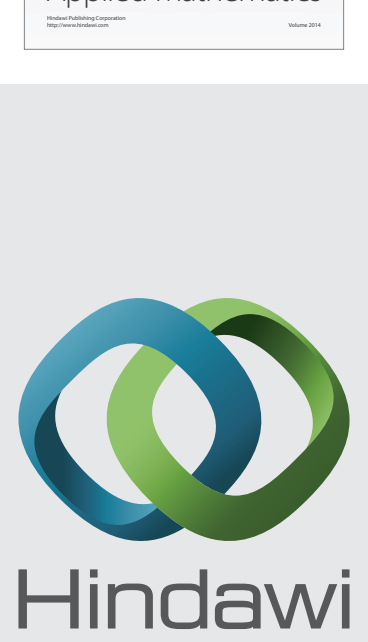

Submit your manuscripts at http://www.hindawi.com
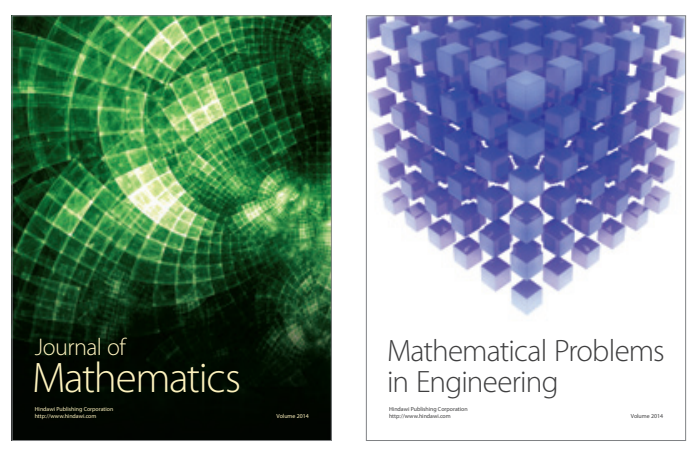

Mathematical Problems in Engineering
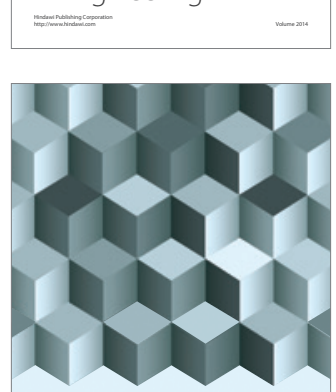

Journal of

Function Spaces
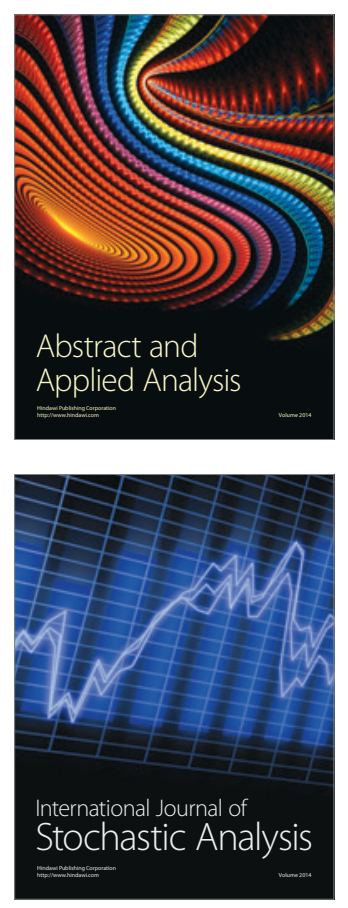

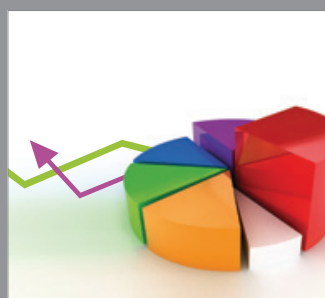

ournal of

Probability and Statistics

Promensencen
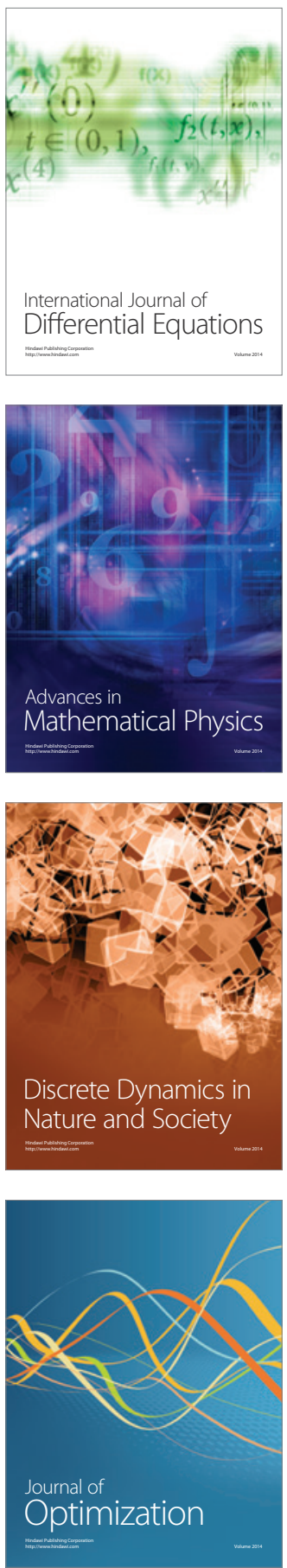